\title{
Chronic aspiration of gastric fluid accelerates pulmonary allograft dysfunction in a rat model of lung transplantation
}

\author{
Matthew G. Hartwig, MD, ${ }^{a}$ James Z. Appel, MD, ${ }^{a}$ Bin Li, MD, PhD, ${ }^{a}$ Chong-Chao Hsieh, MD, ${ }^{a}$ Yong Han Yoon, MD, ${ }^{a}$
} Shu S. Lin, MD, PhD, ${ }^{a}$ William Irish, PhD, ${ }^{b}$ William Parker, $\mathrm{PhD}^{\mathrm{a}}{ }^{\mathrm{a}}$ and R. Duane Davis, $\mathrm{MD}^{\mathrm{a}}$

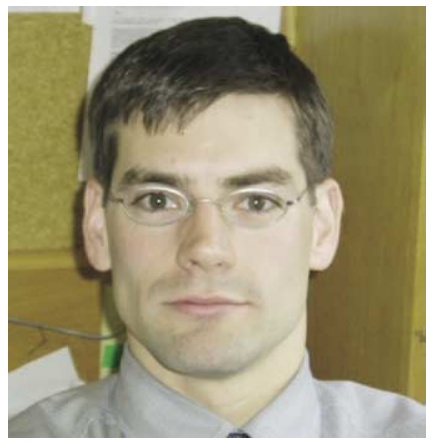

Dr Hartwig

From the Department of Surgery, Duke University, Durham, NC, ${ }^{\text {a }}$ and RTI Health Solutions, ${ }^{\mathrm{b}}$ Research Triangle Park, NC.

Supported in part by an American College of Surgeons Faculty Research Grant (S.S.L.) and National Institutes of Health grant R01 HL60232-03 (D.D.).

The authors have no conflicts of interest with regard to this work.

Read at the Eighty-fifth Annual Meeting of The American Association for Thoracic Surgery, San Francisco, Calif, April 10-13, 2005.

Received for publication April 8, 2005; revisions received June 20, 2005; accepted for publication June 30, 2005

Address for reprints: R. Duane Davis, MD, Department of Surgery, Duke University Medical Center, Box 2605, DUMC, Durham, NC 27710 (E-mail: davis053@mc.duke.edu).

J Thorac Cardiovasc Surg 2006;131:209-17

$0022-5223 / \$ 32.00$

Copyright () 2006 by The American Association for Thoracic Surgery

doi:10.1016/j.jtcvs.2005.06.054
Objective: Emerging clinical evidence suggests that gastroesophageal reflux disease is associated with pulmonary allograft dysfunction. In this study, we used a model of rat lung transplantation to test the hypothesis that chronic aspiration of gastric contents accelerates pulmonary allograft dysfunction.

Methods: We evaluated the effects of chronic aspiration on pulmonary isografts (strain F344) and pulmonary allografts (strain WKY to strain F344). Chronic aspiration consisted of $0.5 \mathrm{~mL} / \mathrm{kg}$ of filtered gastric contents injected weekly into the left lung for 4 to 8 weeks beginning 1 week after transplantation. Seven days after the last aspiration, animals were killed, and grafts were evaluated grossly and by histologic and immunochemical analyses, including Masson trichrome staining for collagen and immunostaining for $\mathrm{CD} 8^{+}$and $\mathrm{CD} 8^{+}$cells. Serum cytokine concentrations were determined by bead-based immunoassays or enzyme-linked immunosorbent assay.

Results: Allografts without aspiration $(\mathrm{n}=12)$ demonstrated a relatively normal architecture with diffuse International Society for Heart and Lung Transplantation grade 3 acute rejection; occasional grade 4 rejection was noted. In contrast, allografts with chronic aspiration $(n=7)$ demonstrated severe grade 4 acute rejection with significant monocyte infiltration, fibrosis, and loss of normal alveolar anatomy. Grossly, 8 (67\%) of 12 allografts without aspiration seemed to inflate and perfuse normally, whereas all allografts exposed to chronic aspiration were firm and shrunken, without the ability to ventilate $(P=.013$; Fisher exact test). Aspiration was associated with increases in graft-infiltrating macrophages and $\mathrm{CD} 8^{+} \mathrm{T}$ cells and higher levels of serum transforming growth factor $\beta$.

Conclusions: Chronic aspiration of gastric contents promotes accelerated allograft failure and may promote a profibrotic environment.

$\mathrm{L}$ ung transplantation represents a viable option for improving the quality of life and extending the survival of end-stage lung disease patients. The primary limiting factor in long-term survival for lung transplant recipients remains bronchiolitis obliterans (OB). ${ }^{1} \mathrm{OB}$ is a pathologic lesion characterized by luminal compromise of small airways by eosinophilic plaques and collagen deposition, but it is very challenging to diagnose by transbronchial biopsy. ${ }^{2}$ Because of the difficulty with diagnosis, a clinical correlate for $\mathrm{OB}$ was developed and is known as bronchiolitis obliterans syndrome, or BOS. ${ }^{3}$ The most important predictor of BOS development is the frequency and severity of acute rejection episodes; however, medical noncompliance, HLA mismatching, and infection may also play a role. $^{4-7}$

A growing number of studies associate gastroesophageal reflux disease (GERD) with various pulmonary pathologies, including interstitial pulmonary fibrosis, ${ }^{8}$ chronic bronchitis, ${ }^{9}$ and asthma. ${ }^{10}$ Also, there is some indication that patients with 


$$
\begin{aligned}
& \text { Abbreviations and Acronyms } \\
& \begin{aligned}
\text { APC } & =\text { antigen-presenting cell } \\
\text { BOS } & =\text { bronchiolitis obliterans syndrome } \\
\text { F344 } & =\text { Fischer F344 } \\
\text { GERD } & =\text { gastroesophageal reflux disease } \\
\text { IL } & =\text { interleukin } \\
\text { OB } & =\text { bronchiolitis obliterans } \\
\text { PA } & =\text { pulmonary artery } \\
\text { PV } & =\text { pulmonary vein } \\
\text { TGF } & =\text { transforming growth factor } \\
\text { WKY } & =\text { Wistar-Kyoto }
\end{aligned}
\end{aligned}
$$

Institutional Animal Care and Use Committee as mandated by the Animal Welfare Act and were in compliance with the Guide for the Care and Use of Laboratory Animals.

\section{Transplantation}

Left lungs from either WKY or F344 rats were orthotopically transplanted into F344 rats via a nonsuturing external cuff technique similar to that described by Mizobuchi and colleagues. ${ }^{17}$ Briefly, the donor was anesthetized with inhaled isoflurane (2\%$3 \%)$ and then administered pentobarbital $(50 \mathrm{mg} / \mathrm{kg})$ intraperitoneally. The donor was then ventilated via a tracheotomy ( 80 breaths per minute; tidal volume of $7 \mathrm{~mL} / \mathrm{kg}$ ), and a midline sternolaparotomy was performed. The animal was heparinized with heparin $(2 \mathrm{U} / \mathrm{g})$ delivered intravenously before flushing of the pulmonary artery (PA) with $25 \mathrm{~mL}$ of Celsior preservation solution (SangStat, Fremont, Calif) at $4{ }^{\circ} \mathrm{C}$. The heart-lung block was then removed, and the left lung was isolated and separated. After procurement, the left pulmonary vein (PV), $\mathrm{PA}$, and main bronchus were passed through polytetrafluoroethylene cuffs, everted over the cuffs, and then secured with 5-0 silk suture. A 16-gauge cuff was used for the PA, whereas the $\mathrm{PV}$ and main bronchus required 14-gauge cuffs. After cuff placement, the donor graft was wrapped in Celsior-soaked gauze and placed on ice.

The recipient rodent was sedated with ketamine $(40 \mathrm{mg} / \mathrm{kg})$ and atropine $(0.01 \mathrm{mg} / \mathrm{kg})$ and then orotracheally intubated with a 14-gauge intravenous catheter. The recipient was anesthetized with isoflurane $(2 \%-3 \%)$ during the procedure and ventilated at a rate of 80 breaths per minute and a tidal volume of $7 \mathrm{~mL} / \mathrm{kg}$. A left thoracotomy was made in the third intercostal space, followed by dissection of the left lung hilum. The left PV, PA, and main bronchus were then isolated and occluded with microaneurysm clamps (Mizuho America, Beverly, Mass), and small incisions were made anteriorly for subsequent insertion of each respective cuff. After insertion, the cuffs were secured by using 5-0 silk suture, and ventilation and perfusion were reestablished by removing the hilar clamps. The chest was closed in 3 layers, and a pleural drainage tube was maintained until the recipient recovered from anesthesia. No immunosuppression was used in any recipient.

\section{Repetitive Aspiration}

Gastric fluid was collected from rodents by using the following procedure: a small midline incision was made over the upper abdomen, and the peritoneal cavity was entered. The stomach and proximal duodenum were dissected free. The proximal duodenum was then ligated with 2-0 silk suture, and a small gastrostomy was created through which a gastrostomy tube was inserted. The gastrostomy tube was placed to gravity drainage and the gastric fluid was collected in a $50-\mathrm{mL}$ sterile conical tube. The gastric fluid was then filtered through a $70-\mu \mathrm{m}$ strainer (BD Biosciences, Bedford, Mass) to remove particulate matter, pooled, and frozen for future aspiration experiments. The gastric fluid $\mathrm{pH}$ was 2 to 2.5 just before intratracheal instillation.

Each recipient in the aspiration arm of the experiment began receiving once-weekly gastric fluid instillation into the left lung 1 week after transplantation. To accomplish this, each recipient was sedated and orotracheally intubated with a 16-gauge intravenous catheter. The subject was then placed with the head directed 
upward at a $35^{\circ}$ to $40^{\circ}$ angle in the left lateral decubitus position. A small silicone rubber catheter was inserted into the distal trachea through which $0.5 \mathrm{~mL} / \mathrm{kg}$ of gastric fluid was injected into the left lung. The small silicone rubber catheter and the endotracheal tube were then flushed clear with air. Experiments were terminated, and animals were killed 5 or 9 weeks after transplantation-1 week after the final aspiration event.

On the basis of previous work in the field of aspiration pneumonitis, in addition to unpublished preliminary experiments in our laboratory, the dose of aspirated material was determined to be approximately $10 \%$ to $50 \%$ of a potentially lethal bolus of gastric secretions. ${ }^{18,19}$ This dose of gastric fluid was initially selected to avoid early mortality as a result of aspiration. In our preliminary experiments, we experienced no mortality as a direct result of the aspiration event. Images of rat lungs containing various amounts (0-0.5 mL) of aspirated contrast material were quantitatively assessed by using the program Scion Image (Scion Corporation, Frederick, Md). A linear relationship $(r>0.99)$ between image density and aspirated material was obtained for the left lung, whereas no increase in image density was observed in the right (untreated) lungs of the same rats. With this method, approximately $99 \%$ of instilled contrast administered in this manner selectively enters the left lung (data not shown).

\section{Histology}

Lung tissue was fixed by using $2 \%$ paraformaldehyde. Left and right lung biopsy specimens were stained with hematoxylin and eosin as well as Masson trichrome stains. The extent of fibrosis in trichrome-stained specimens was graded by a single pathologist in a blinded fashion by using a continuous numeric scale in which the overall severity of interstitial fibrosis was determined on a scale of 0 (normal lung) to 8 (total fibrous obliteration), as described elsewhere. ${ }^{20}$ Immunohistochemical analysis was performed with anti-rat CD68 and CD8 monoclonal antibodies (Becton Dickinson, Franklin Lakes, NJ). Anti-rat CD68 recognizes a single-chain glycoprotein of $110 \mathrm{kd}$ that is expressed predominantly on the lysosomal membrane of myeloid cells. Weak cell-surface expression also occurs. The antigen is expressed by most tissue macrophages and is expressed weakly by peripheral blood granulocytes. ${ }^{21}$ Anti-rat CD8 recognizes the rat CD8 cell-surface antigen expressed by a subset of $\mathrm{T}$ lymphocytes, most thymocytes, and most natural killer cells. ${ }^{22}$

\section{Cytokine Assays}

Serum specimens were thawed and cytokine concentrations of interleukin (IL)-2, IL-4, IL-6, IL-10, and tumor necrosis factor $\alpha$ were measured by using Bio-Plex multiplex bead-based immunoassays (Bio-Rad Laboratories, Hercules, Calif). Specimens were analyzed by using a Luminex 100 flow-based, dual-laser array reader (Luminex, Austin, Tex), and cytokine concentrations were quantified by using Bio-Plex Manager Software (Bio-Rad Laboratories). The concentration of transforming growth factor (TGF)- $\beta 1$ was determined from samples by using an enzyme-linked immunosorbent assay-based sandwich immunoassay (R\&D Systems, Minneapolis, Minn).

\section{Statistics}

Continuous data are presented as the mean \pm SEM or median and interquartile range (25th and 75 th percentiles), and categorical data
TABLE 1. Description of lung transplant groups

\begin{tabular}{lr}
\hline Experimental groups* & No. subje \\
\hline 5-wk isotransplantation† & 6 \\
5-wk isotransplantation with aspiration & 4 \\
5-wk allotransplantation & 6 \\
5-wk allotransplantation with aspiration & 4 \\
9-wk allotransplantation & 6 \\
9-wk allotransplantation with aspiration & $3 \S$ \\
All allotransplantations (5- and 9-wk groups) & 12 \\
All allotransplantations (5- and 9-wk groups) & 7 \\
$\quad$ with aspiration &
\end{tabular}

*Time given indicates how many weeks after transplantation that the recipient was killed. †lsotransplantations involved strains F344 to F344. $\ddagger$ Allotransplantations involved strains WKY to F344. §One recipient from the 9-week allotransplantation with aspiration was lost secondary to a technical complication during an episode of aspirate instillation.

are presented as counts and percentages. Between-group comparisons were made by using the Wilcoxon rank sum test, a nonparametric equivalent to the standard 2-sample $t$ test, for continuoustype data, and Fisher exact tests were used for categorical-type data. All analyses were performed with SAS for Windows software version 8.0 (SAS Institute, Cary, NC).

\section{Results}

\section{Effect of Chronic Aspiration on the Gross Pathology of Pulmonary Grafts}

Nineteen allotransplantations were performed by using grafts of WKY donor origin in F344 recipients. Seven (37\%) of these recipients received directed intratracheal gastric fluid instillation as described in "Materials and Methods." Ten isotransplantations (F344 to F344) were performed in a similar fashion; 4 (40\%) of these animals received directed intratracheal gastric fluid instillation. Thus, 12 allotransplantation cases and 6 isotransplantation cases were not administered gastric contents and served as control groups (Table 1). Upon termination of the experiment, $8(67 \%)$ of 12 of the allotransplant recipients without aspiration had functional grafts according to gross examination of perfusion, mechanical ventilation, color, and size (Figure 1, inset). In contrast, all 7 allograft recipients receiving gastric fluid aspiration demonstrated diminutive, noncompliant, and discolored grafts without normal perfusion (Fisher exact test; $P=.013$ ). Consistent with the idea that organ dysfunction was associated with an allospecific response, isografts in the face of aspiration seemed functional at the termination of the experiment.

\section{Effect of Chronic Aspiration on the Histologic Appearance of Pulmonary Grafts}

Representative histologic characteristics of pulmonary grafts from the 4 groups of recipients are depicted in Figure 2. Allotransplant recipients receiving no aspiration developed moderate interstitial mononuclear cell infiltrates with mod- 


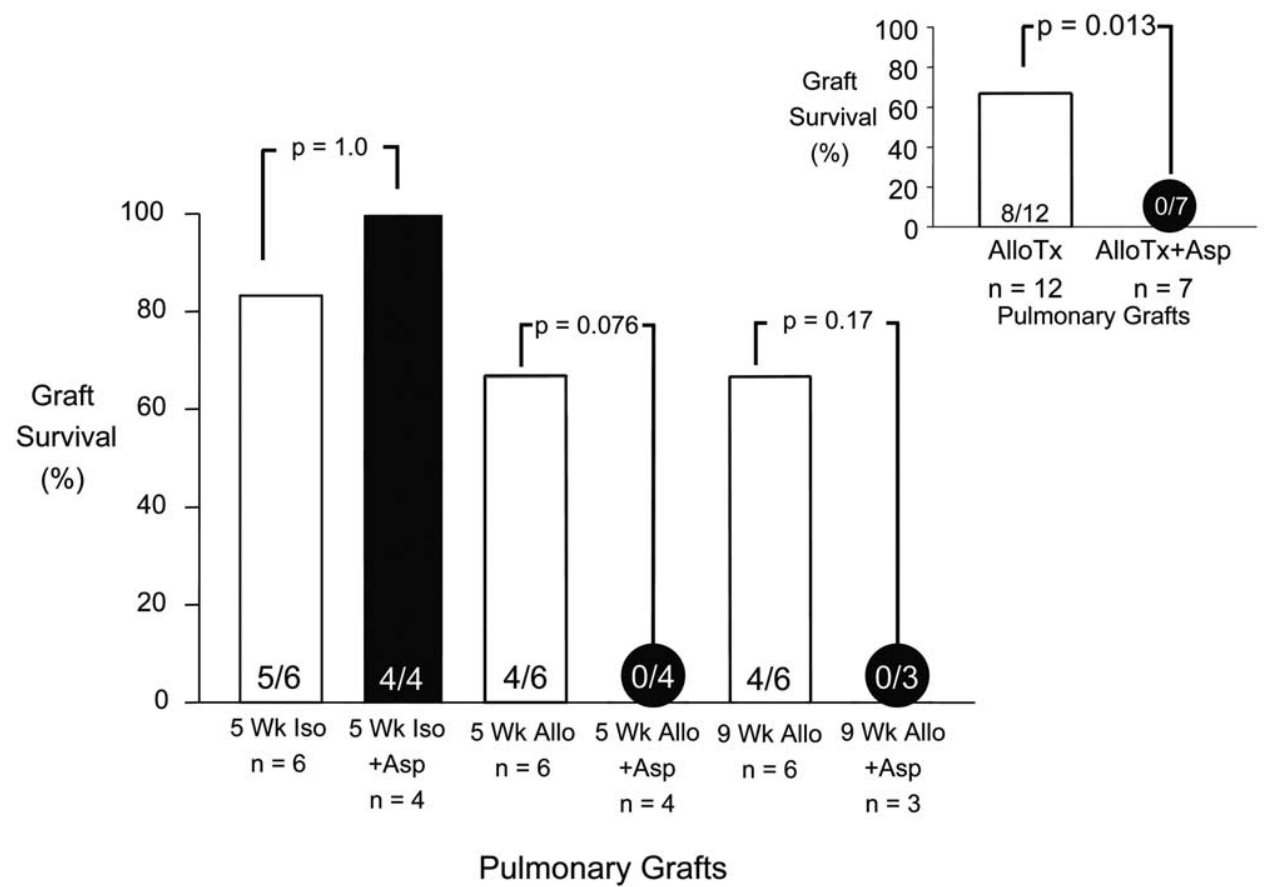

Figure 1. Effect of chronic aspiration on isograft and allograft survival. The percentage of functioning organs in rats receiving pulmonary isografts or allografts with or without gastric fluid aspiration is shown. The inset provides a compilation of 5- and 9-week allotransplantation results. None of the allotransplant organs receiving aspirated gastric fluid were functioning at the termination of the experiment. Statistical comparisons were performed with Fisher exact tests. Allo or AlloTx, Allotransplant; Iso, isotransplant; +Asp, with aspiration.

erate to severe acute rejection (grade A3 to A4) but maintained relatively normal lung architecture. Conversely, pulmonary allografts exposed to chronic aspiration demonstrated considerably worse histologic injury, with extensive interstitial and alveolar infiltration of mononuclear cells (grade A4). These recipients also exhibited diffuse destruction of alveolar and interstitial structure. Microscopic examination of isotransplant grafts with or without aspiration did not reveal acute rejection or abnormal parenchymal architecture.

Control allografts lacking aspiration demonstrated moderate amounts of interstitial fibrosis, with a median fibrotic grade of 1.63 (interquartile range, 1.46-5.20; Figure 3, inset). However, the allografts receiving aspiration revealed a more severe fibrotic pattern, with a median fibrotic grade of 6.33 (interquartile range, $3.75-8.00 ; P=.016$; Figure 3 ).
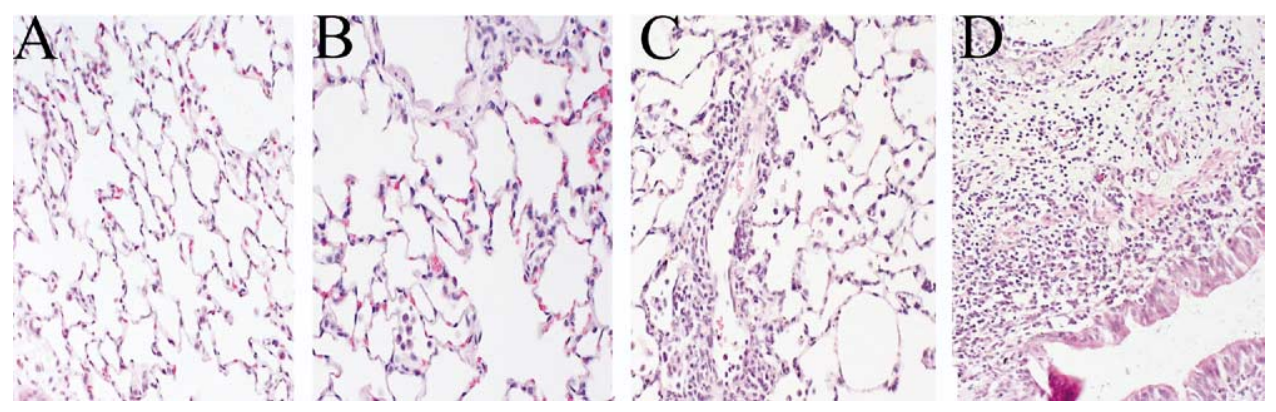

Figure 2. Effect of chronic aspiration on the histologic characteristics of isografts and allografts. Lung tissue was procured from rats receiving isografts ( $A$ and $B$ ) or allografts ( $C$ and $D)$ with (B and $D$ ) or without ( $A$ and $C$ ) gastric fluid aspiration. Tissues were fixed, processed, and stained with hematoxylin and eosin as described in "Materials and Methods." 


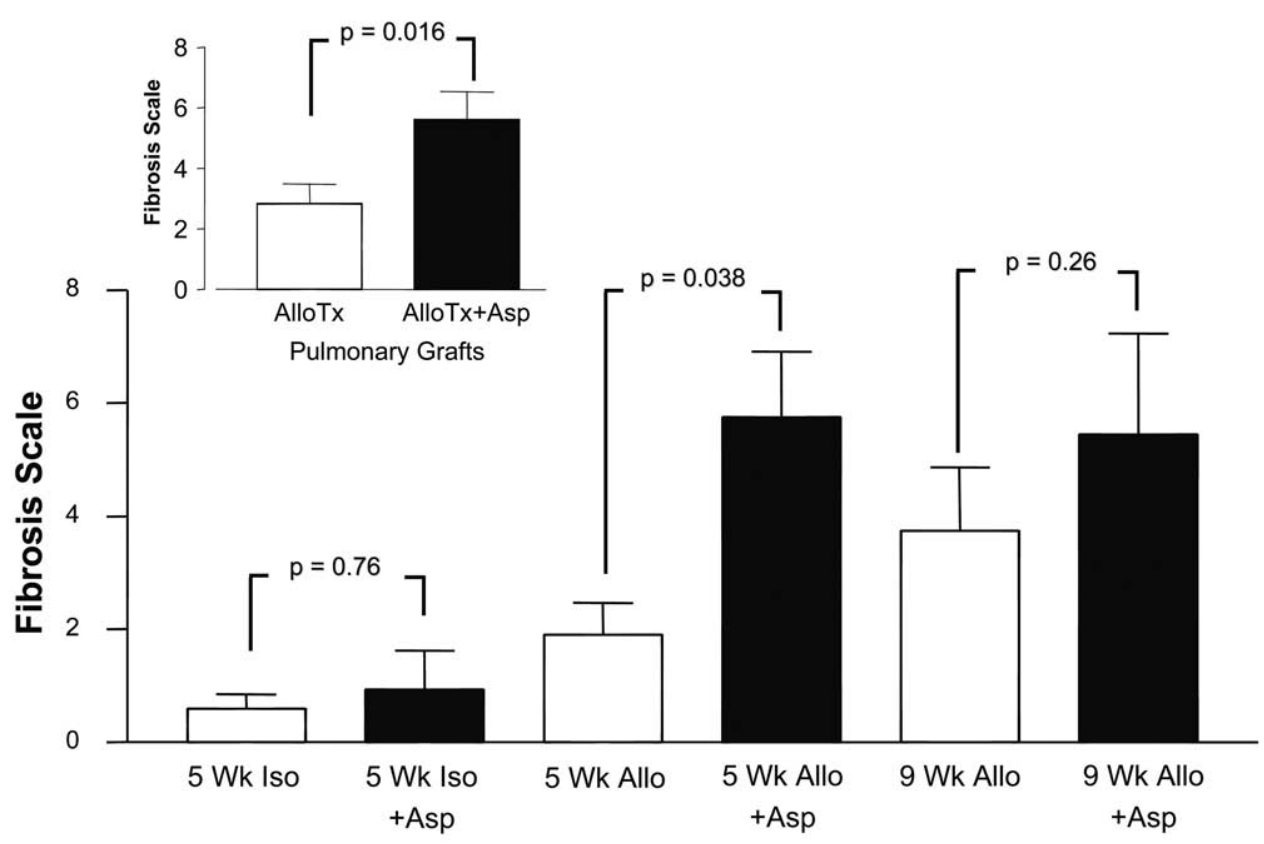

Pulmonary Grafts

Figure 3. Effect of chronic aspiration on fibrosis in isografts and in allografts. Lung tissue was procured from rats receiving isografts or allografts with or without gastric fluid aspiration. Tissues were fixed, processed, and stained with Masson trichrome stain as described in "Materials and Methods." The fibrosis score shown was determined as described in "Materials and Methods" and was compared between groups by using the Wilcoxon rank sum test. The inset provides a compilation of 5- and 9-week allotransplantation results. Allo or AlloTx, Allotransplant; Iso, isotransplant; +Asp, with aspiration.

Without aspiration, the 5-week allotransplants (median fibrotic grade, 1.55; interquartile range, 1.05-3.14) had less fibrosis than the 9-week allotransplants (median fibrotic grade, 3.85; interquartile range, 1.04-6.37; $P=.24$ ). However, with aspiration, the 5-week allotransplants (median fibrotic grade, 5.65; range, 3.71-8.00) had levels of fibrosis similar to those of the 9-week allotransplants (median fibrotic grade, 6.33; range, 2.00-8.00; $P=.86$ ). Overall, control isografts and isografts from recipients with aspiration appeared normal, without significant fibrosis (Figure 3).

\section{Cellular Infiltrates in Response to Chronic Aspiration in Pulmonary Grafts}

As described previously, substantial mononuclear cell infiltrates were observed in allografts but not in isografts. Although mononuclear infiltrates were observed in the allografts of animals with gastric fluid aspiration and in those with no aspiration, the infiltrates were more pronounced in those with gastric fluid aspiration. Consistent with these observations, isografts demonstrated minimal numbers of $\mathrm{CD}^{+} 8^{+}$or $\mathrm{CD}^{+}$cells by immunohistochemical analysis, regardless of the presence or absence of aspiration (Figure 4).
Conversely, immunohistochemical analysis revealed significant $\mathrm{CD} 68^{+}$and $\mathrm{CD}^{+}$cellular infiltrates in allografts; the most severe infiltration by $\mathrm{CD}^{+} 8^{+}$and $\mathrm{CD} 8^{+}$cells occurred in allografts with aspiration. $\mathrm{CD}^{+} 8^{+}$and $\mathrm{CD}^{+}$cells in the allografts with aspiration were present diffusely, throughout the alveolar spaces and interstitium, and their presence did not seem to correlate with areas of perivascular infiltration or cuffing.

\section{Serum Cytokines}

Figure 5 shows the serum levels of TGF- $\beta$ in the different transplantation groups at the termination of the experiment. Whereas control allografts without aspiration had levels of TGF- $\beta$ similar to those in isografts, regardless of how long after transplantation they were, allografts with aspiration had significantly higher levels of TGF- $\beta$ (Figure 5; $P=$ .026). Similarly, the isografts with aspiration also had greater serum concentrations of TGF- $\beta$ than control isografts; however, this difference did not reach statistical difference (Figure 5; $P=.34$ ). Serum concentrations of IL-4 were not detectable in any of the groups, whereas IL-2, IL-6, IL-10, 


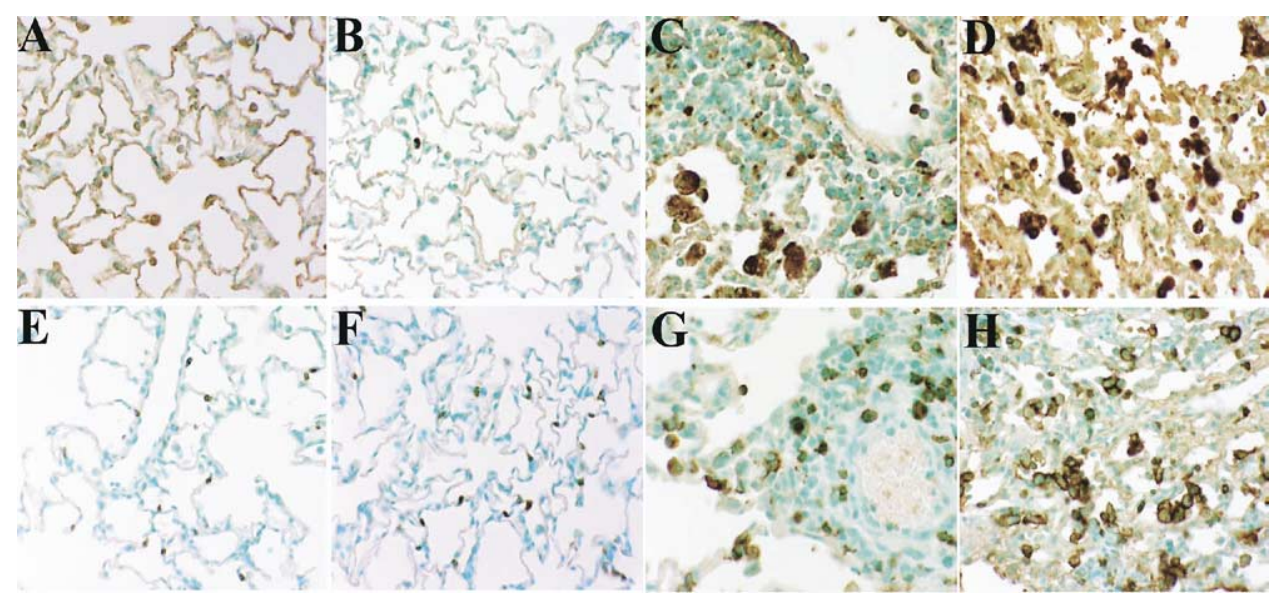

Figure 4. Immunohistochemical analysis of cellular infiltrates in isografts and allografts, both with and without chronic aspiration. Lung tissue was procured from rats receiving isografts $(A, B, E$, and $F)$ or allografts $(C, D, G$, and H) with (B, D, F, and H) or without (A, C, E, and G) gastric fluid aspiration. Tissues were stained for either CD68 (A-D) or CD8 (E-H) as described in "Materials and Methods."

and tumor necrosis factor $\alpha$ serum levels were detected but did not differ significantly in the allotransplantation animals secondary to the administration of gastric fluid (data not shown).

\section{Discussion}

Several groups have emphasized the role that nonimmune injury may have in accelerating pulmonary allograft dysfunction. Possible nonimmune insults include cytomegalovirus pneumonitis, viral infection, ischemia-reperfusion injury, and GERD. ${ }^{4,5,12,23,24}$ Previous clinical studies by our group supported a relationship between GERD and premature chronic graft dysfunction. ${ }^{12}$ Initial case reports suggested that microaspiration events secondary to excessive GERD may be contributing to graft failure. ${ }^{25}$ After this, our group reported impaired pulmonary function in lung recipients with GERD that could be improved with a surgical corrective procedure for GERD (ie, fundoplication). ${ }^{12,14}$ Reviews of our lung transplant database also suggested an association between GERD and more severe acute rejection that may be ameliorated with fundoplication. ${ }^{13}$ This study describes a novel animal model for chronic aspiration in a rodent pulmonary transplant system that was designed to study the cellular and molecular mechanisms underlying accelerated graft dysfunction secondary to aspiration of enteric contents.

Early animal models of acute lung injury secondary to aspiration, or Mendelson syndrome, demonstrated that a $\mathrm{pH}$ of less than 2.5 was critical for promoting lung injury. ${ }^{26}$ Using a swine model of aspiration, others showed that pepsin also significantly contributed to pulmonary injury and that instillation of large, potentially lethal quantities of gastric fluid led to significant alterations in pulmonary physiology and subsequent pulmonary fibrosis. ${ }^{27}$ Although important, the aforementioned studies do not address the role of GERD in lung transplantation for multiple reasons. First, the earlier studies focused on a single large-volume aspiration event. However, in lung recipients, GERD-induced injury is likely secondary to repetitive smaller aspiration events that may not create overt symptoms or aberrant physiology. Although this has not been demonstrably proven in human lung recipients, preliminary data show high levels of pepsin and bile acids in bronchoalveolar lavage samples of lung recipients without a history of known aspiration events. ${ }^{15,16}$ This study is the first to describe the effects of smaller, repetitive, and nonlethal doses of gastric contents aspirated into a pulmonary isograft or allograft.

Data demonstrating that aspiration leads to accelerated loss of graft function corroborate findings from retrospective reviews of human clinical data. In this study, all of the allografts that received aspirated gastric contents were nonfunctioning by the end of the study, compared with only $33 \%$ of the control allografts. This significant difference in graft function seems to involve allospecific, immune-mediated lung injury, because aspiration had no such effect on isografts. In addition, the survival of isografts in the face of aspiration eliminates technical failure as a potential cause for the loss of allograft function. Consistent with the conclusion that allospecific immune reactions are involved in the aspiration-associated allograft failure we observed is the observation that early works in aspiration injury demonstrated deleterious physiologic effects from low $\mathrm{pH}$ and pepsin, but not lung failure. ${ }^{26,27}$ Likewise, our model of chronic aspiration, when performed for up to 4 months in 


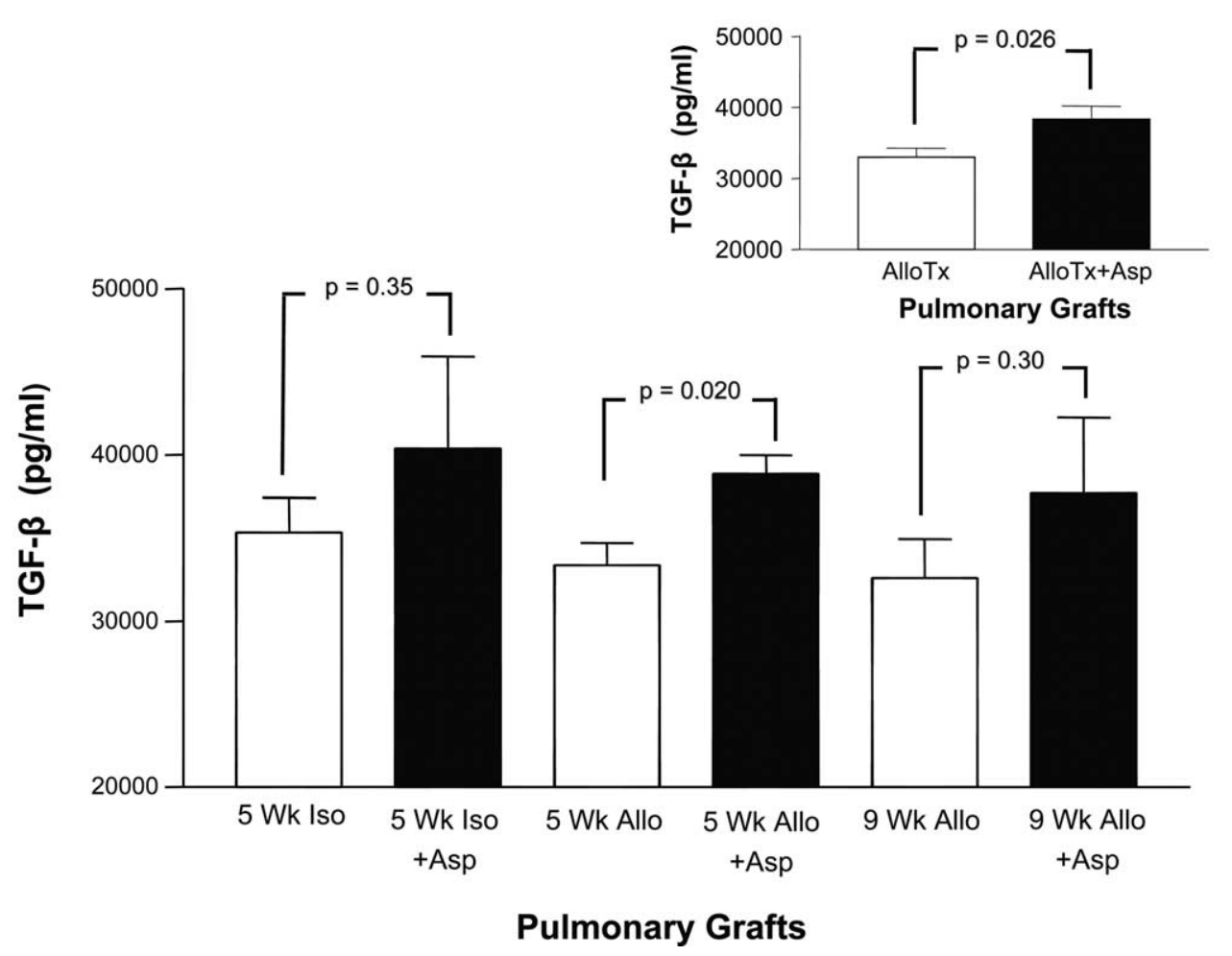

Figure 5. Effect of chronic aspiration on the levels of TGF- $\beta$ in the serum of isograft and allograft recipients. The level of TGF- $\beta$ in the serum of rats receiving isografts or allografts with or without gastric fluid aspiration is shown. The inset provides a compilation of 5- and 9-week allotransplantation results. Comparisons between groups were made by using an unpaired 2-tailed $t$ test. Allo or AlloTx, Allotransplant; Iso, isotransplant; + Asp, with aspiration.

nontransplant rats, did not lead to overt pulmonary failure in any of the animals (J.Z.A. and M.G.H., unpublished data). Therefore, some level of baseline alloimmune injury seems to play an important role in aspiration-induced loss of graft function.

OB is thought to develop secondary to alloimmune injury that manifests clinically as acute rejection. ${ }^{28}$ Acute rejection is believed to occur as a result of antigen-presenting cells (APCs) processing and presenting donor antigen to host $\mathrm{T}$ lymphocytes. APCs are responsible for initiating and propagating acute rejection, whereas graft-infiltrating lymphocytes mediate the rejection response. This study demonstrates an increase in graft infiltration by macrophages and $\mathrm{CD}^{+}$lymphocytes as a result of aspiration. These results are consistent with a predilection for acute rejection as a result of aspiration. Alveolar macrophages are important sources of chemotactic agents for lymphocytes during rejection, and depleting those macrophages downregulates

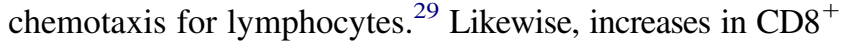
cells correlate with the progression of acute rejection in a model of rat transplantation. ${ }^{30}$ Taken together, these results suggest that aspiration of gastric contents leads to an increased potential for antigen presentation secondary to in- creases in graft APCs, as well as increased rejection injury as a result of more graft-infiltrating lymphocytes.

TGF- $\beta$ is a potent profibrotic cytokine with a multifaceted role in lung transplantation. Recently, reports suggest that TGF- $\beta$ may play an important role in the pathogenesis of OB. Elssner and colleagues ${ }^{31}$ demonstrated increased levels of TGF- $\beta$ in epithelial lining fluid and in the bronchoalveolar lavage fluid cells of patients with BOS. In a murine heterotopic tracheal model of $\mathrm{OB}$, expression of TGF- $\beta$ was increased in obliterated grafts compared with unaffected isografts. ${ }^{32}$ Additionally, immunohistochemistry analysis of biopsy samples from human lung recipients demonstrated greater TGF- $\beta$ expression in patients with $\mathrm{OB}$ than in those without OB. ${ }^{33}$ Finally, inhibition of TGF- $\beta$ intracellular signaling pathways in tracheal allografts decreased intraluminal deposition of fibrous material. ${ }^{34}$ The current study is consistent with previous research in that it associates TGF- $\beta$ with pulmonary allograft fibrosis. This study suggests that one pathway for aspiration-induced lung injury may be via promoting a profibrotic cytokine milieu that, when combined with a host alloimmune response, leads to accelerated graft failure. It also demonstrates that chronic aspiration is capable of increasing systemic TGF- $\beta$ 
expression, as opposed to changes limited to the pulmonary tissue.

\section{References}

1. Trulock EP, Edwards LB, Taylor DO, Boucek MM, Keck BM, Hertz MI. The registry of the International Society for Heart and Lung Transplantation: twenty-first official adult heart transplant report2004. J Heart Lung Transplant. 2004;23:804-15.

2. Yousem SA, Berry GJ, Cagle PT, et al. Revision of the 1990 working formulation for the classification of pulmonary allograft rejection: Lung Rejection Study Group. J Heart Lung Transplant. 1996;15(1 Pt 1): $1-15$.

3. Cooper JD, Billingham M, Egan T, et al. A working formulation for the standardization of nomenclature and for clinical staging of chronic dysfunction in lung allografts. International Society for Heart and Lung Transplantation. J Heart Lung Transplant. 1993;12:713-6.

4. Bando K, Paradis IL, Similo S, et al. Obliterative bronchiolitis after lung and heart-lung transplantation. An analysis of risk factors and management. J Thorac Cardiovasc Surg. 1995;110:4-13; discussion 14.

5. Kroshus TJ, Kshettry VR, Savik K, John R, Hertz MI, Bolman RM III. Risk factors for the development of bronchiolitis obliterans syndrome after lung transplantation. J Thorac Cardiovasc Surg. 1997;114:195202.

6. Girgis RE, Tu I, Berry GJ, et al. Risk factors for the development of obliterative bronchiolitis after lung transplantation. J Heart Lung Transplant. 1996;15:1200-8.

7. Schulman LL, Weinberg AD, McGregor C, Galantowicz ME, SuciuFoca NM, Itescu S. Mismatches at the HLA-DR and HLA-B loci are risk factors for acute rejection after lung transplantation. Am J Respir Crit Care Med. 1998;157(6 Pt 1):1833-7.

8. Raghu G. The role of gastroesophageal reflux in idiopathic pulmonary fibrosis. Am J Med. 2003;115(Suppl 3A):60S-4S.

9. Ducolone A, Vandevenne A, Jouin H, et al. Gastroesophageal reflux in patients with asthma and chronic bronchitis. Am Rev Respir Dis. 1987; 135:327-32.

10. Vincent D, Cohen-Jonathan AM, Leport J, et al. Gastro-oesophageal reflux prevalence and relationship with bronchial reactivity in asthma. Eur Respir J. 1997;10:2255-9.

11. Bowrey DJ, Peters JH, DeMeester TR. Gastroesophageal reflux disease in asthma: effects of medical and surgical antireflux therapy on asthma control. Ann Surg. 2000;231:161-72.

12. Davis RD Jr, Lau CL, Eubanks S, et al. Improved lung allograft function after fundoplication in patients with gastroesophageal reflux disease undergoing lung transplantation. J Thorac Cardiovasc Surg. 2003; 125:533-42.

13. Hartwig MG, Cantu E, Appel JZ, Woreta H, Palmer SM, Davis RD. Non-alloimmune injury mediated by gastroesophageal reflux precipitates alloimmune injury in lung transplant patients. J Heart Lung Transplant. In press.

14. Cantu E, Appel JZ, Hartwig MG, et al. Early fundoplication prevents chronic allograft dysfunction in patients with gastroesophageal reflux disease. Ann Thorac Surg. 2004;78:1142-51.

15. Ward C, Brownlee IA, Forrest I, et al. Pepsin like activity in BAL is evidence of gastric aspiration in lung allografts. In: American Thoracic Society 100th International Conference, 2004, Orlando, Fla. 2004.

16. D'Ovidio F, Mura M, Waddell TK, et al. Acids in bronchoalveolar lavage after lung transplantation as a marker of pulmonary aspiration associated with alveolar neutrophilia. In: International Society for Heart and Lung Transplantation Annual Meeting, 2004, San Francisco, Calif. 2004.

17. Mizobuchi T, Sekine Y, Yasufuku K, Fujisawa T, Wilkes DS. Comparison of surgical procedures for vascular and airway anastomoses that utilize a modified non-suture external cuff technique for experimental lung transplantation in rats. J Heart Lung Transplant. 2004;23:889-93.

18. Hamelberg W, Bosomworth PP. Aspiration pneumonitis: experimental studies and clinical observations. Anesth Analg. 1964;43:669-77.

19. Greenfield LJ, Singleton RP, McCaffree DR, Coalson JJ. Pulmonary effects of experimental graded aspiration of hydrochloric acid. Ann Surg. 1969;170:74-86.
20. Ashcroft T, Simpson JM, Timbrell V. Simple method of estimating severity of pulmonary fibrosis on a numerical scale. J Clin Pathol. 1988;41:467-70.

21. Damoiseaux JG, Dopp EA, Calame W, Chao D, MacPherson GG, Dijkstra CD. Rat macrophage lysosomal membrane antigen recognized by monoclonal antibody. Immunology. 1994;83:140-7.

22. Brideau RJ, Carter PB, McMaster WR, Mason DW, Williams AF. Two subsets of rat $\mathrm{T}$ lymphocytes defined with monoclonal antibodies. Eur J Immunol. 1980;10:609-15.

23. Billings JL, Hertz MI, Savik K, Wendt $\mathrm{CH}$. Respiratory viruses and chronic rejection in lung transplant recipients. J Heart Lung Transplant. 2002;21:559-66.

24. Garantziotis S, Howell DN, McAdams HP, Davis RD, Henshaw NG, Palmer SM. Influenza pneumonia in lung transplant recipients: clinical features and association with bronchiolitis obliterans syndrome. Chest. 2001;119:1277-80.

25. Palmer SM, Miralles AP, Howell DN, Brazer SR, Tapson VF, Davis RD. Gastroesophageal reflux as a reversible cause of allograft dysfunction after lung transplantation. Chest. 2000;118:1214-7.

26. Vandam LD. Aspiration of gastric contents in the operative period. N Engl J Med. 1965;273:1206-8.

27. Popper H, Juettner F, Pinter J. The gastric juice aspiration syndrome (Mendelson syndrome). Aspects of pathogenesis and treatment in the pig. Virchows Arch A Pathol Anat Histopathol. 1986;409:105-17.

28. Boehler A, Estenne M. Post-transplant bronchiolitis obliterans. Eur Respir J. 2003;22:1007-18.

29. Sekine Y, Yasufuku K, Heidler KM, et al. Monocyte chemoattractant protein-1 and RANTES are chemotactic for graft infiltrating lymphocytes during acute lung allograft rejection. Am J Respir Cell Mol Biol. 2000;23:719-26.

30. Takehisa Y, Sakiyama S, Uyama T, et al. Progressive increase of $\mathrm{CD} 4(+) / \mathrm{CD} 45 \mathrm{RC}(-)$ lymphocytes after allograft rat lung transplantation: a marker of acute rejection. J Thorac Cardiovasc Surg. 2002; 124:675-83.

31. Elssner A, Jaumann F, Dobmann S, et al. Elevated levels of interleukin-8 and transforming growth factor-beta in bronchoalveolar lavage fluid from patients with bronchiolitis obliterans syndrome: proinflammatory role of bronchial epithelial cells. Munich Lung Transplant Group. Transplantation. 2000;70:362-7.

32. Aris RM, Walsh S, Chalermskulrat W, Hathwar V, Neuringer IP. Growth factor upregulation during obliterative bronchiolitis in the mouse model. Am J Respir Crit Care Med. 2002;166:417-22.

33. El-Gamel A, Sim E, Hasleton P, et al. Transforming growth factor beta (TGF-beta) and obliterative bronchiolitis following pulmonary transplantation. J Heart Lung Transplant. 1999;18:828-37.

34. Ramirez AM, Takagawa S, Sekosan M, Jaffe HA, Varga J, Roman J. Smad3 deficiency ameliorates experimental obliterative bronchiolitis in a heterotopic tracheal transplantation model. Am J Pathol. 2004; 165:1223-32.

\section{Discussion}

Dr Raphael Bueno (Boston, Mass). In this elegant study, you propose and evaluate a new animal model to study the effect of reflux in lung grafts in rats. You compared the effect of injecting gastric contents into lung allograft versus lung isograft, showing more rapid progression of fibrosis in allografts receiving acid. You conclude that aspirated gastric secretions enhance acute rejection type injuries, which would likely eventually lead to chronic rejection. Certainly we have heard from your group over the years how this is relevant in lung transplantation.

I have several brief questions. First, you used a $0.5 \mathrm{cc} / \mathrm{kg}$ injection transtracheally of reflux material once a week. How did you come up with that dose? Did you do dose-response tests? If you translate it to humans, that's $35 \mathrm{cc}$ 's for the $70-\mathrm{kg}$ person, and that's a lot.

Dr Hartwig. That is correct. What we did was a combination of literature search and our own informal LD50 [median lethal 
dose] experiments essentially. If you look at some of the earlier work in aspiration injury, also referred to as Mendelson syndrome, you will note that it's usually a 1-time instillation of acid and it's usually a very, very large dose, 10 to 20 times what we were using, and occasionally they would see death of the animals subsequent to that. We knew we wanted to be below any sort of lethal level, so we performed our own informal LD50 experiments and determined that we would be well below that, probably about a tenth of that dose. I don't know if you can directly correlate rats and humans as far as lung volume and instillation of volumes. If you did a milliliter per kilogram for a human, it would be much more than you would expect on a microaspiration level, but, again, since this was sort of a novel model, we wanted to make sure that we were giving enough to see an injury response pattern in the time frames and time constraints that we had for the experiment while making sure we stayed well below any sort of lethal dose, and we did not experience any lethal events during the injection of the acid.

Dr Bueno. Certainly in humans we give immunosuppression after transplantation. Have you tried or are you proposing to try adding immunosuppression in the allograft group to see how that will influence the progression of disease?

Dr Hartwig. Absolutely. I think that's probably the primary limiting factor of this study. Our baseline rejection injury in the non-aspirating animals was significant, much more so than we had hoped and thought originally. So I think it's going to be very important to not only minimize that baseline injury from the standpoint of injury without aspiration, but then in order to be able to extend the experiment out more months and better recreate what we see clinically, the next step is going to be immunosuppression of the animals. Those projects are in process. Unfortunately, we would like to take them out 2, 3, or 4 months to better characterize that. It takes time to do those experiments.

Dr Bueno. And, finally, you were very careful in the types of rats you transplanted and treated, but in the manuscript you made available to me, you just collected the gastric secretions from rodents. You didn't specify the type. We're dealing with potentially immunologic injuries that you are suggesting or proposing based on the mononuclear infiltrates, et cetera. Do you think that the source of protein-whether it is from the same rat type or a different rat type-that comes from the stomach has any effect on what happens, or is it just an initial chemical reaction?

Dr Hartwig. Right. That's a wonderful question. When we first started this work, it's not something that we had planned for originally, and so we were arbitrarily using gastric fluid from either WKY or F344 rats. However, for the next set of experiments, what we did prospectively was collect gastric fluid only from the F344 rodents and collected it all in advance, pooled it, and kept it together, so that there will be homogeneous gastric fluid in the next set of experiments.

\section{Targeted}

The Journal of Thoracic and Cardiovascular Surgery gives you two tables of contents. The condensed table of contents tells you at a glance what topics and authors are presented each month. The expanded table of contents gives you a brief abstract of each article. You select only those articles of most interest to you for further reading. 